\title{
Relativistic third-order dissipative fluid dynamics from kinetic theory
}

\author{
Amaresh Jaiswal \\ Tata Institute of Fundamental Research, Homi Bhabha Road, Mumbai 400005, India
}

(Dated: August 19, 2018)

\begin{abstract}
We present the derivation of a novel third-order hydrodynamic evolution equation for shear stress tensor from kinetic theory. Boltzmann equation with relaxation time approximation for the collision term is solved iteratively using Chapman-Enskog like expansion to obtain the nonequilibrium phasespace distribution function. Subsequently, the evolution equation for shear stress tensor is derived from its kinetic definition up-to third-order in gradients. We quantify the significance of the new derivation within one-dimensional scaling expansion and demonstrate that the results obtained using third-order viscous equations derived here provides a very good approximation to the exact solution of Boltzmann equation in relaxation time approximation. We also show that the time evolution of pressure anisotropy obtained using our equations is in better agreement with transport results when compared with an existing third-order calculation based on the second-law of thermodynamics.
\end{abstract}

PACS numbers: 05.20.Dd, 47.75+f, 47.10.-g, 47.10.A-

Fluid dynamics is an effective theory describing the long-wavelength limit of the microscopic dynamics of a system. While nonrelativistic fluid dynamics finds applications in various aspects of physics and engineering, the domain of applicability of relativistic fluid dynamics is in the field of astrophysics, cosmology and high-energy heavy-ion collisions. The collective behavior of the hot and dense matter (which is believed to have existed in the very early universe) created in ultra-relativistic heavy-ion collisions has been studied quite extensively within the framework of relativistic fluid dynamics. To study the evolution of a hydrodynamic system, it is natural to first employ the equations of ideal fluid dynamics. However, as ideal fluids are hypothetical by virtue of uncertainty principle 1], the dissipative effects can not be ignored.

Relativistic dissipative fluid dynamics is formulated as an order-by-order expansion in gradients, ideal hydrodynamics being zeroth-order. The first-order theories, collectively known as relativistic Navier-Stokes (NS) theory, are due to Eckart [2] and Landau-Lifshitz [3]. However, these theories involve parabolic differential equations and suffer from acausality and numerical instability. The second-order theory by Israel and Stewart (IS) [4] with its hyperbolic equations solves the acausality problem [5] but may not guarantee stability. Despite the success of IS theory in explaining a wide range of collective phenomena observed in heavy-ion collisions, its formulation is based on strong assumptions and approximations. The original IS theory derived from Boltzmann equation (BE) uses two powerful assumptions in the derivation of dissipative equations: use of second moment of $\mathrm{BE}$ and the 14moment approximation [4, [6]. In Ref. [7], although the dissipative equations were derived directly from their definitions without resorting to second-moment of BE, however the 14-moment approximation was still employed. In Ref. [8] it was shown that both these assumptions are unnecessary and instead of 14-moment approximation, iterative solution of $\mathrm{BE}$ was used to obtain the dissipative evolution equations from their definitions.

Apart from these problems in the formulation, IS the- ory suffers from several other shortcomings. In onedimensional Bjorken scaling expansion [9], for large viscosities or small initial time, IS theory has resulted in unphysical effects such as reheating of the expanding medium [10] and negative longitudinal pressure [11]. Further, the scaling solutions of IS equations when compared with transport results show disagreement for $\eta / s>0.5$ indicating the breakdown of second-order theory [ 5,12 . With this motivation, in Ref. 13], second-order dissipative equations were derived from $\mathrm{BE}$ where the collision term was generalized to include nonlocal effects via gradients of the distribution function. Moreover, in Refs. [8, 14] it was demonstrated that a heuristic inclusion of higher-order corrections led to an improved agreement with transport results. In fact, the derivation of higherorder constitutive equations from kinetic theory for nonrelativistic systems has been known for a long time [15]. Thus it is of interest to improvise the relativistic secondorder theory by incorporating higher-order corrections.

In this Rapid Communication, we derive a new relativistic third-order evolution equation for shear stress tensor from kinetic theory. Without resorting to the widely used Grad's 14-moment approximation [6], we iteratively solve the $\mathrm{BE}$ in relaxation time approximation (RTA) to obtain nonequilibrium phase-space distribution function. We subsequently derive equation of motion for shear stress tensor up-to third-order, directly from its definition. Within one-dimensional scaling expansion, the results obtained using third-order evolution equations derived here shows improved agreement with exact solution of BE as compared to second-order equations. We also demonstrate that the evolution of pressure anisotropy obtained using our equations shows better agreement with the transport results as compared to those obtained by using an existing third-order equation derived from entropy considerations.

The hydrodynamic evolution of a system is governed by the conservation equations for energy and momentum. The conserved energy-momentum tensor can be expressed in terms of single-particle, phase-space distri- 
bution function and tensor decomposed into hydrodynamic variables [16]. For a system of massless particles, bulk viscosity vanishes leading to

$$
T^{\mu \nu}=\int d p p^{\mu} p^{\nu} f(x, p)=\epsilon u^{\mu} u^{\nu}-P \Delta^{\mu \nu}+\pi^{\mu \nu},
$$

where $d p \equiv g d \mathbf{p} /\left[(2 \pi)^{3}|\mathbf{p}|\right], g$ being the degeneracy factor, $p^{\mu}$ is the particle four-momentum and $f(x, p)$ is the phase-space distribution function. In the tensor decompositions, $\epsilon, P$ and $\pi^{\mu \nu}$ are respectively energy density, pressure and the shear stress tensor. The projection operator $\Delta^{\mu \nu} \equiv g^{\mu \nu}-u^{\mu} u^{\nu}$ is orthogonal to the hydrodynamic four-velocity $u^{\mu}$ defined in the Landau frame: $T^{\mu \nu} u_{\nu}=\epsilon u^{\mu}$. The metric tensor is Minkowskian, $g^{\mu \nu} \equiv \operatorname{diag}(+,-,-,-)$.

Energy-momentum conservation, $\partial_{\mu} T^{\mu \nu}=0$ yields the fundamental evolution equations for $\epsilon$ and $u^{\mu}$

$$
\begin{aligned}
\dot{\epsilon}+(\epsilon+P) \theta-\pi^{\mu \nu} \nabla_{(\mu} u_{\nu)} & =0 \\
(\epsilon+P) \dot{u}^{\alpha}-\nabla^{\alpha} P+\Delta_{\nu}^{\alpha} \partial_{\mu} \pi^{\mu \nu} & =0 .
\end{aligned}
$$

We use the notation $\dot{A} \equiv u^{\mu} \partial_{\mu} A$ for comoving derivative, $\theta \equiv \partial_{\mu} u^{\mu}$ for the expansion scalar, $A^{(\alpha} B^{\beta)} \equiv$ $\left(A^{\alpha} B^{\beta}+A^{\beta} B^{\alpha}\right) / 2$ for symmetrization and $\nabla^{\alpha} \equiv \Delta^{\mu \alpha} \partial_{\mu}$ for space-like derivative. In the massless limit, the energy density and pressure are related as $\epsilon=3 P \propto \beta^{-4}$. The inverse temperature, $\beta \equiv 1 / T$, is defined by the Landau matching condition $\epsilon=\epsilon_{0}$ where $\epsilon_{0}$ is the equilibrium energy density. In this limit, Eqs. (2) can be used to obtain the derivatives of $\beta$ as

$$
\dot{\beta}=\frac{\beta}{3} \theta-\frac{\beta}{12 P} \pi^{\rho \gamma} \sigma_{\rho \gamma}, \quad \nabla^{\alpha} \beta=-\beta \dot{u}^{\alpha}-\frac{\beta}{4 P} \Delta_{\rho}^{\alpha} \partial_{\gamma} \pi^{\rho \gamma},
$$

where $\sigma^{\rho \gamma} \equiv \nabla^{(\rho} u^{\gamma)}-(\theta / 3) \Delta^{\rho \gamma}$ is the velocity stress tensor. The above identities will be helpful in the derivation of shear evolution equation.

The expression for shear stress tensor $\left(\pi^{\mu \nu}\right)$ can be obtained in terms of the out-of-equilibrium part of the distribution function. To this end, we write the nonequilibrium distribution function as $f=f_{0}+\delta f$, where the deviation from equilibrium is assumed to be small $(\delta f \ll f)$. The equilibrium distribution function represents Boltzmann statistics of massless particles at vanishing chemical potential, $f_{0}=\exp (-\beta u \cdot p)$, where $u \cdot p \equiv u_{\mu} p^{\mu}$. From Eq. (1), $\pi^{\mu \nu}$ can be expressed in terms of $\delta f$ as

$$
\pi^{\mu \nu}=\Delta_{\alpha \beta}^{\mu \nu} \int d p p^{\alpha} p^{\beta} \delta f
$$

where $\Delta_{\alpha \beta}^{\mu \nu} \equiv \Delta_{(\alpha}^{\mu} \Delta_{\beta)}^{\nu}-(1 / 3) \Delta^{\mu \nu} \Delta_{\alpha \beta}$ is a traceless symmetric projection operator orthogonal to $u^{\mu}$. To proceed further, the form of $\delta f$ has to be specified. In the following, Boltzmann equation in RTA will be solved iteratively to obtain $\delta f$ order-by-order in gradients.

Nonequilibrium phase-space distribution function can be obtained by solving the one-body kinetic equation such as the Boltzmann equation. The most common technique of generating solutions to such equations is the Chapman-Enskog expansion where the particle distribution function is expanded about its equilibrium value in powers of space-time gradients [17]

$$
f=f_{0}+\delta f, \quad \delta f=\delta f^{(1)}+\delta f^{(2)}+\cdots,
$$

where $\delta f^{(1)}$ is first-order in derivatives, $\delta f^{(2)}$ is secondorder and so on. Subsequently, the relativistic Boltzmann equation with relaxation time approximation for the collision term [18],

$$
p^{\mu} \partial_{\mu} f=-u \cdot p \frac{\delta f}{\tau_{R}} \Rightarrow f=f_{0}-\left(\tau_{R} / u \cdot p\right) p^{\mu} \partial_{\mu} f,
$$

can be solved iteratively as $[8,19]$

$$
f_{1}=f_{0}-\frac{\tau_{R}}{u \cdot p} p^{\mu} \partial_{\mu} f_{0}, \quad f_{2}=f_{0}-\frac{\tau_{R}}{u \cdot p} p^{\mu} \partial_{\mu} f_{1}, \quad \cdots
$$

where $f_{n}=f_{0}+\delta f^{(1)}+\delta f^{(2)}+\cdots+\delta f^{(n)}$. To first and second-order in derivatives, we obtain

$$
\begin{aligned}
\delta f^{(1)} & =-\frac{\tau_{R}}{u \cdot p} p^{\mu} \partial_{\mu} f_{0}, \\
\delta f^{(2)} & =\frac{\tau_{R}}{u \cdot p} p^{\mu} p^{\nu} \partial_{\mu}\left(\frac{\tau_{R}}{u \cdot p} \partial_{\nu} f_{0}\right) .
\end{aligned}
$$

The above expressions for nonequilibrium part of the distribution function along with Eq. (4) will be used in the derivation of shear evolution equations.

As a side remark, note that the RTA for the collision term, $C[f]=-(u \cdot p) \delta f / \tau_{R}$ in Eq. (6), should satisfy current and energy-momentum conservation, i.e., the zeroth and first moment of the collision term should vanish [16]. Assuming the relaxation time $\tau_{R}$ to be independent of momenta, these conservation equations are satisfied only if the fluid four-velocity is defined in the Landau frame [18]. Hence, within RTA, the Landau frame is imposed and is not a choice.

The first-order expression for shear stress tensor can be obtained from Eq. (4) using $\delta f=\delta f^{(1)}$ from Eq. (8),

$$
\pi^{\mu \nu}=\Delta_{\alpha \beta}^{\mu \nu} \int d p p^{\alpha} p^{\beta}\left(-\frac{\tau_{R}}{u \cdot p} p^{\mu} \partial_{\mu} f_{0}\right) .
$$

Using Eqs. (33) and keeping only those terms which are first-order in gradients, the integrals in the above equation reduce to

$$
\pi^{\mu \nu}=2 \tau_{R} \beta_{\pi} \sigma^{\mu \nu}, \quad \beta_{\pi}=\frac{4}{5} P .
$$

To obtain the second-order evolution equation, we follow the methodology discussed in Ref. 7]. The evolution of the shear stress tensor can be obtained by considering the comoving derivative of Eq. (4),

$$
\dot{\pi}^{\langle\mu \nu\rangle}=\Delta_{\alpha \beta}^{\mu \nu} \int d p p^{\alpha} p^{\beta} \delta \dot{f},
$$

where the notation $A^{\langle\mu \nu\rangle} \equiv \Delta_{\alpha \beta}^{\mu \nu} A^{\alpha \beta}$ represents traceless symmetric projection orthogonal to $u^{\mu}$. 
The comoving derivative of the nonequilibrium part of the distribution function $(\delta \dot{f})$ can be obtained by rewriting Eq. (6) in the form

$$
\delta \dot{f}=-\dot{f}_{0}-\frac{1}{u \cdot p} p^{\gamma} \nabla_{\gamma} f-\frac{\delta f}{\tau_{R}},
$$

Using this expression for $\delta \dot{f}$ in Eq. (12), we obtain

$$
\dot{\pi}^{\langle\mu \nu\rangle}+\frac{\pi^{\mu \nu}}{\tau_{R}}=-\Delta_{\alpha \beta}^{\mu \nu} \int d p p^{\alpha} p^{\beta}\left(\dot{f}_{0}+\frac{1}{u \cdot p} p^{\gamma} \nabla_{\gamma} f\right) .
$$

It is clear that in the above equation, the Boltzmann relaxation time $\tau_{R}$ can be replaced by the shear relaxation time $\tau_{\pi}$. By comparing the first-order evolution Eq. (11) with the relativistic Navier-Stokes equation $\pi^{\mu \nu}=2 \eta \sigma^{\mu \nu}$, the shear relaxation time is obtained in terms of the firstorder transport coefficient, $\tau_{\pi}=\eta / \beta_{\pi}$.

Note that for the shear evolution equations to be second-order in gradients, the distribution function on the right hand side of Eq. (14) need to be computed only till first-order, i.e., $f=f_{1}=f_{0}+\delta f^{(1)}$. Using Eq. (8) for $\delta f^{(1)}$ and Eqs. (3) for derivatives of $\beta$, and keeping terms upto quadratic order in gradients, the second-order shear evolution equation is obtained as [8]

$\dot{\pi}^{\langle\mu \nu\rangle}+\frac{\pi^{\mu \nu}}{\tau_{\pi}}=2 \beta_{\pi} \sigma^{\mu \nu}+2 \pi_{\gamma}^{\langle\mu} \omega^{\nu\rangle \gamma}-\frac{10}{7} \pi_{\gamma}^{\langle\mu} \sigma^{\nu\rangle \gamma}-\frac{4}{3} \pi^{\mu \nu} \theta$,

where $\omega^{\mu \nu} \equiv\left(\nabla^{\mu} u^{\nu}-\nabla^{\nu} u^{\mu}\right) / 2$ is the vorticity tensor. We have used the first-order expression for shear stress tensor, Eq. (11), to replace $\sigma^{\mu \nu} \rightarrow \pi^{\mu \nu}$ such that the relaxation times appearing on the right hand side of Eq. (14) are absorbed.

To derive a third-order evolution equation for shear stress tensor, the distribution function on the right hand side of Eq. (14) needs to be computed till second-order $\left(\delta f=\delta f^{(1)}+\delta f^{(2)}\right)$. In order to account for all the higher-order terms, Eq. (15) was used to substitute for $\sigma^{\mu \nu}$. Employing Eqs. (3) for derivatives of $\beta$ and keeping terms upto cubic order in derivatives, we finally obtain a unique third-order evolution equation for shear stress tensor after a straightforward but tedious algebra

$$
\begin{aligned}
\dot{\pi}^{\langle\mu \nu\rangle}= & -\frac{\pi^{\mu \nu}}{\tau_{\pi}}+2 \beta_{\pi} \sigma^{\mu \nu}+2 \pi_{\gamma}^{\langle\mu} \omega^{\nu\rangle \gamma}-\frac{10}{7} \pi_{\gamma}^{\langle\mu} \sigma^{\nu\rangle \gamma} \\
& -\frac{4}{3} \pi^{\mu \nu} \theta+\frac{25}{7 \beta_{\pi}} \pi^{\rho\langle\mu} \omega^{\nu\rangle \gamma} \pi_{\rho \gamma}-\frac{1}{3 \beta_{\pi}} \pi_{\gamma}^{\langle\mu} \pi^{\nu\rangle \gamma} \theta \\
& -\frac{38}{245 \beta_{\pi}} \pi^{\mu \nu} \pi^{\rho \gamma} \sigma_{\rho \gamma}-\frac{22}{49 \beta_{\pi}} \pi^{\rho\langle\mu} \pi^{\nu\rangle \gamma} \sigma_{\rho \gamma} \\
& -\frac{24}{35} \nabla^{\langle\mu}\left(\pi^{\nu\rangle \gamma} \dot{u}_{\gamma} \tau_{\pi}\right)+\frac{4}{35} \nabla^{\langle\mu}\left(\tau_{\pi} \nabla_{\gamma} \pi^{\nu\rangle \gamma}\right) \\
& -\frac{2}{7} \nabla_{\gamma}\left(\tau_{\pi} \nabla^{\langle\mu} \pi^{\nu\rangle \gamma}\right)+\frac{12}{7} \nabla_{\gamma}\left(\tau_{\pi} \dot{u}^{\langle\mu} \pi^{\nu\rangle \gamma}\right) \\
& -\frac{1}{7} \nabla_{\gamma}\left(\tau_{\pi} \nabla^{\gamma} \pi^{\langle\mu \nu\rangle}\right)+\frac{6}{7} \nabla_{\gamma}\left(\tau_{\pi} \dot{u}^{\gamma} \pi^{\langle\mu \nu\rangle}\right) \\
& -\frac{2}{7} \tau_{\pi} \omega^{\rho\langle\mu} \omega^{\nu\rangle \gamma} \pi_{\rho \gamma}-\frac{2}{7} \tau_{\pi} \pi^{\rho\langle\mu} \omega^{\nu\rangle \gamma} \omega_{\rho \gamma} \\
& -\frac{10}{63} \tau_{\pi} \pi^{\mu \nu} \theta^{2}+\frac{26}{21} \tau_{\pi} \pi_{\gamma}^{\langle\mu} \omega^{\nu\rangle \gamma} \theta .
\end{aligned}
$$

This is the main result of the present work. We note that Eq. (16) represents only a subset of all possible third order terms because bulk viscosity and heat current has been neglected.

We compare the third-order shear evolution equation derived in the present work with that obtained in Ref. [14]. In the latter work, the shear evolution equation was derived by invoking second law of thermodynamics from kinetic definition of entropy four-current, expanded till third-order in $\pi^{\mu \nu}$. For ease of comparison, we write the evolution equation obtained in Ref. [14] in the form

$$
\begin{aligned}
\dot{\pi}^{\langle\mu \nu\rangle}= & -\frac{\pi^{\mu \nu}}{\tau_{\pi}^{\prime}}+2 \beta_{\pi}^{\prime} \sigma^{\mu \nu}-\frac{4}{3} \pi^{\mu \nu} \theta+\frac{5}{36 \beta_{\pi}^{\prime}} \pi^{\mu \nu} \pi^{\rho \gamma} \sigma_{\rho \gamma} \\
& -\frac{16}{9 \beta_{\pi}^{\prime}} \pi_{\gamma}^{<\mu} \pi^{\nu>\gamma} \theta,
\end{aligned}
$$

where $\beta_{\pi}^{\prime}=2 P / 3$ and $\tau_{\pi}^{\prime}=\eta / \beta_{\pi}^{\prime}$. We observe that the right-hand-side of Eq. (17) contains one second-order and two third-order terms compared to three secondorder and fourteen third-order terms obtained in the present work, i.e., Eq. (16). It is well known that the approach based on entropy method fails to capture all the terms in the dissipative evolution equations even at second-order. Moreover, the discrepancy at third-order confirms the fact that the evolution equation obtained by invoking second law of thermodynamics is incomplete.

To demonstrate the numerical significance of the thirdorder shear evolution equation derived here, we consider boost-invariant Bjorken expansion of a system consisting of massless Boltzmann gas [9]. Working in Milne coordinates $\left(\tau, x, y, \eta_{s}\right)$, where $\tau=\sqrt{t^{2}-z^{2}}, \eta_{s}=\tanh ^{-1}(z / t)$, and with $u^{\mu}=(1,0,0,0)$, we observe that only the $\eta_{s} \eta_{s}$ component of Eq. (16) survives. In this scenario, $\omega^{\mu \nu}=\dot{u}^{\mu}=\nabla^{\mu} \tau_{\pi}=0, \theta=1 / \tau$ and $\sigma^{\eta_{s} \eta_{s}}=-2 /\left(3 \tau^{3}\right)$. Defining $\pi \equiv-\tau^{2} \pi^{\eta_{s} \eta_{s}}$, we find that $\pi^{\rho \gamma} \sigma_{\rho \gamma}=\pi / \tau$, and

$$
\begin{aligned}
& \dot{\pi}^{\left\langle\eta_{s} \eta_{s}\right\rangle}=-\frac{1}{\tau^{2}} \frac{d \pi}{d \tau}, \pi_{\gamma}^{\left\langle\eta_{s}\right.} \sigma^{\left.\eta_{s}\right\rangle \gamma}=-\frac{\pi}{3 \tau^{3}}, \pi_{\gamma}^{\left\langle\eta_{s}\right.} \pi^{\left.\eta_{s}\right\rangle \gamma}=-\frac{\pi^{2}}{2 \tau^{2}}, \\
& \pi^{\rho\left\langle\eta_{s}\right.} \pi^{\left.\eta_{s}\right\rangle \gamma} \sigma_{\rho \gamma}=-\frac{\pi^{2}}{2 \tau^{3}}, \quad \nabla^{\left\langle\eta_{s}\right.} \nabla_{\gamma} \pi^{\left.\eta_{s}\right\rangle \gamma}=\frac{2 \pi}{3 \tau^{4}}, \\
& \nabla_{\gamma} \nabla^{\left\langle\eta_{s}\right.} \pi^{\left.\eta_{s}\right\rangle \gamma}=\frac{4 \pi}{3 \tau^{4}}, \quad \nabla^{2} \pi^{\left\langle\eta_{s} \eta_{s}\right\rangle}=\frac{4 \pi}{3 \tau^{4}} .
\end{aligned}
$$

Using the above results, evolution of $\epsilon$ and $\pi$ from Eqs. (21) and (16) reduces to

$$
\begin{aligned}
& \frac{d \epsilon}{d \tau}=-\frac{1}{\tau}(\epsilon+P-\pi), \\
& \frac{d \pi}{d \tau}=-\frac{\pi}{\tau_{\pi}}+\beta_{\pi} \frac{4}{3 \tau}-\lambda \frac{\pi}{\tau}-\chi \frac{\pi^{2}}{\beta_{\pi} \tau} .
\end{aligned}
$$

The term with coefficient $\chi$ in the above equation contains correction only due to third-order. The firstorder shear expression, $\pi=4 \beta_{\pi} \tau_{\pi} / 3 \tau$, has been used to rewrite some of the third-order contributions in the form $\pi^{2} /\left(\beta_{\pi} \tau\right)$. The transport coefficients in our calculation simplify to

$$
\tau_{\pi}=\frac{\eta}{\beta_{\pi}}, \quad \beta_{\pi}=\frac{4 P}{5}, \quad \lambda=\frac{38}{21}, \quad \chi=\frac{72}{245} .
$$




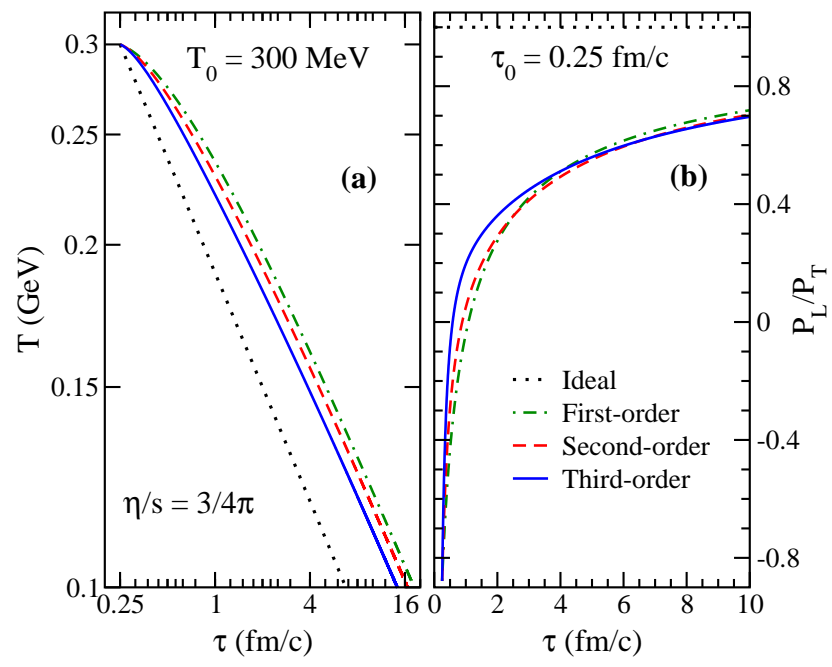

FIG. 1: (Color online) Time evolution of (a) temperature and (b) pressure anisotropy $\left(P_{L} / P_{T}\right)$, in ideal (dotted line), first-order (dashed-dotted lines), second-order (dashed line) and third-order (solid lines) hydrodynamics, for Navier-Stokes initial condition, $\left(\pi_{0}=4 \eta / 3 \tau_{0}\right)$.

We compare these transport coefficients with those obtained from Eq. (17), where they reduce to

$$
\tau_{\pi}^{\prime}=\frac{\eta}{\beta_{\pi}^{\prime}}, \quad \beta_{\pi}^{\prime}=\frac{2 P}{3}, \quad \lambda^{\prime}=\frac{4}{3}, \quad \chi^{\prime}=\frac{3}{4} .
$$

For comparison, we also state the exact solution of Eq. (6) in one-dimensional scaling expansion [20, 21]:

$$
f(\tau)=D\left(\tau, \tau_{0}\right) f_{\mathrm{in}}+\int_{\tau_{0}}^{\tau} \frac{d \tau^{\prime}}{\tau_{R}\left(\tau^{\prime}\right)} D\left(\tau, \tau^{\prime}\right) f_{0}\left(\tau^{\prime}\right),
$$

where, $f_{\text {in }}$ and $\tau_{0}$ is the initial distribution function and proper time respectively, and

$$
D\left(\tau_{2}, \tau_{1}\right)=\exp \left[-\int_{\tau_{1}}^{\tau_{2}} \frac{d \tau^{\prime \prime}}{\tau_{R}\left(\tau^{\prime \prime}\right)}\right] .
$$

The damping function, $D\left(\tau_{2}, \tau_{1}\right)$, has the properties $D(\tau, \tau)=1, D\left(\tau_{3}, \tau_{2}\right) D\left(\tau_{2}, \tau_{1}\right)=D\left(\tau_{3}, \tau_{1}\right)$, and

$$
\frac{\partial D\left(\tau_{2}, \tau_{1}\right)}{\partial \tau_{2}}=-\frac{D\left(\tau_{2}, \tau_{1}\right)}{\tau_{R}\left(\tau_{2}\right)}
$$

To obtain the exact solution, the Boltzmann relaxation time is taken to be the same as the shear relaxation time $\left(\tau_{R}=\tau_{\pi}\right)$. The hydrodynamic quantities can then be calculated by using Eq. (23) for the distribution function in Eq. (11) and performing the integrations numerically.

To quantify the differences between ideal, first-order, second-order, and third-order theories, we solve the evolution equations with initial temperature $T_{0}=300 \mathrm{MeV}$ at initial time $\tau_{0}=0.25 \mathrm{fm} / \mathrm{c}$. These values correspond to the Relativistic Heavy-Ion Collider initial conditions [22]. Figure 1 shows proper time evolution of temperature and pressure anisotropy $P_{L} / P_{T} \equiv(P-\pi) /(P+\pi / 2)$

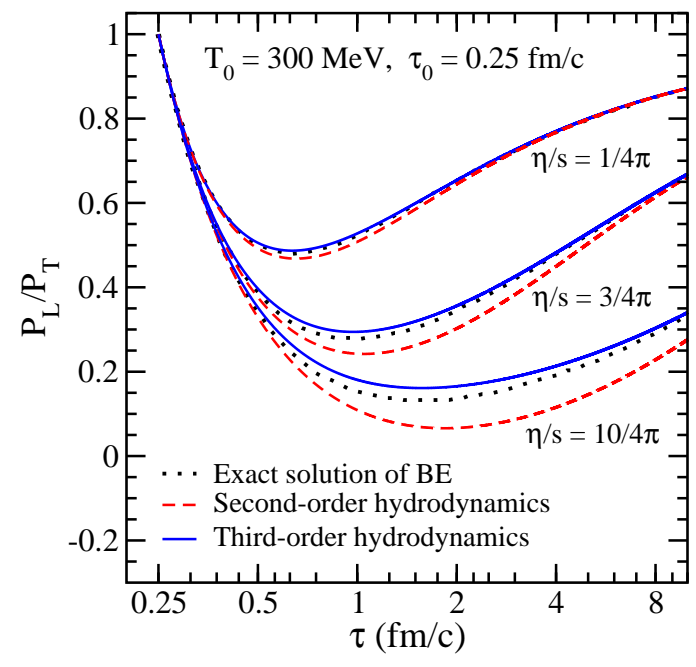

FIG. 2: (Color online) Time evolution of $P_{L} / P_{T}$ obtained using exact solution of Boltzmann equation (dotted line), second-order equations (dashed lines), and third-order equations (solid lines), for isotropic initial pressure configuration $\left(\pi_{0}=0\right)$ and various $\eta / s$.

in ideal (dotted line), first-order (dashed-dotted lines), second-order (dashed line) and third-order (solid lines) hydrodynamics. Here we have assumed Navier-Stokes initial condition for shear pressure $\left(\pi_{0}=4 \eta / 3 \tau_{0}\right)$ and solved the evolution equations for a representative shear viscosity to entropy density ratio, $\eta / s=3 / 4 \pi$.

In Fig. 1 (a), we observe that while ideal hydrodynamics predicts a rapid cooling of the system, evolution based on third-order equation also shows faster temperature drop compared to first-order and second-order evolutions. This implies that the thermal photon and dilepton spectra, which are sensitive to temperature evolution, may be suppressed by including third-order corrections. Moreover, with third-order evolution, the freeze-out temperature is attained at an earlier time which may affect the hadronic spectra as well. In Fig. 1 (b), note that at early times the third-order evolution results in faster isotropization of pressure anisotropy compared to firstorder and second-order. However at later time, the pressure anisotropy obtained using second and third-order equations merge indicating the convergence of gradient expansion in fluid dynamics.

Figure 2. shows the proper time dependence of pressure anisotropy for various $\eta / s$ values with isotropic initial pressure configuration, i.e., $\pi_{0}=0$. The improved agreement of third-order results (solid lines) with the exact solution of BE (dotted line) as compared to second-order results (dashed line) also suggests the convergence of the derivative expansion in hydrodynamics.

Figure [3 also shows the time evolution of pressure anisotropy for initial temperature $T_{0}=500 \mathrm{MeV}$ at initial time $\tau_{0}=0.4 \mathrm{fm} / \mathrm{c}$ which corresponds to Large Hadron Collider initial conditions [22]. The initial pressure configuration is assumed to be isotropic and the evo- 


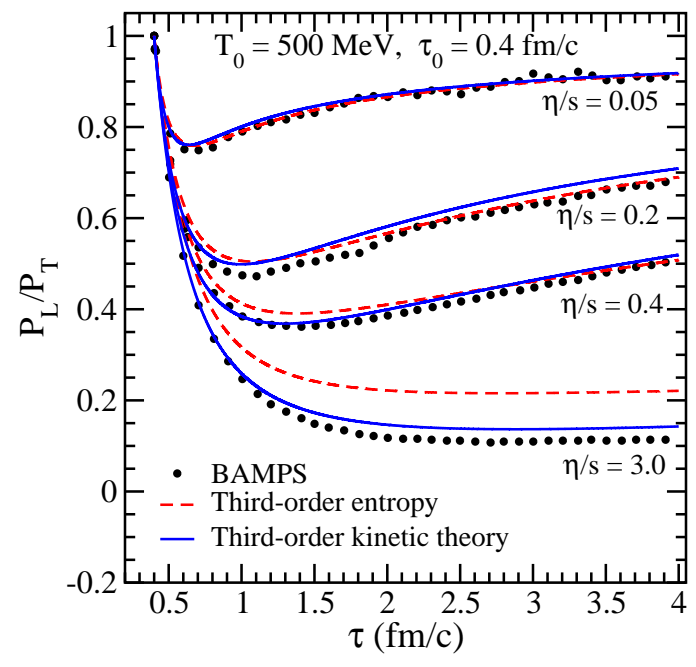

FIG. 3: (Color online) Time evolution of $P_{L} / P_{T}$ in BAMPS (dots), third-order calculation from entropy method, Eq. (17) (dashed lines), and the present work (solid lines), for isotropic initial pressure configuration $\left(\pi_{0}=0\right)$ and various $\eta / s$.

lution is shown for various $\eta / s$ values. The solid lines represent the results obtained in the present work by solving Eqs. (19) and (20) with transport coefficients of Eq. (21). The dashed lines corresponds to results of another third-order theory derived based on second-law of thermodynamics with transport coefficients given in Eq. (22). The dots represent the results of numerical solution of BE using a transport model, the parton cascade BAMPS [14, 23]. The calculations in BAMPS are performed by changing the cross section such that $\eta / s$ remains constant. While the results from entropy derivation overestimate the pressure anisotropy for $\eta / s>0.2$, those obtained in the present work (kinetic theory) are in better agreement with the BAMPS results.

The RTA for the collision term in BE is based on the assumption that the effect of the collisions is to exponentially restore the distribution function to its local equilibrium value. Although the information about the mi- croscopic interactions of the constituent particles is not retained here, it is a reasonably good approximation to describe a system which is close to local equilibrium. It is important to note that although the third-order viscous equations derived here uses BE with RTA for the collision term, the evolution shows good quantitative agreement with BAMPS results which employs realistic collision kernel [23]. Indeed in Ref. 24], it has been shown that for a purely gluonic system at weak coupling and hadron gas with large momenta, BE in RTA is a fairly accurate description. Furthermore, the experimentally observed $1 / \sqrt{m_{T}}$ scaling of the HBT radii, which was shown to be broken by including viscous corrections to the distribution function [25], can be restored by using the form of the non-equilibrium distribution function obtained here [26]. All these factors clearly suggests that the BE in RTA can be applied quite successfully in understanding the hydrodynamic behavior of the strongly interacting matter formed in heavy-ion collisions.

To summarize, we have derived a novel third-order evolution equation for the shear stress tensor from kinetic theory within relaxation time approximation. Instead of Grad's 14-moment approximation, iterative solution of Boltzmann equation was used for the nonequilibrium distribution function and the evolution equation for shear tensor is derived directly from its definition. Within onedimensional scaling expansion, we have demonstrated that the third-order hydrodynamics derived here provides a very good approximation to the exact solution of Boltzmann equation in relaxation time approximation. Our results also show a better agreement with the parton cascade BAMPS for the $P_{L} / P_{T}$ evolution compared to those obtained from entropy derivation.

\section{Acknowledgments}

The author thanks Rajeev S. Bhalerao and Subrata Pal for helpful discussions, and Jasmine Sethi for a critical reading of the manuscript.
[1] P. Danielewicz and M. Gyulassy, Phys. Rev. D 31, 53 (1985).

[2] C. Eckart, Phys. Rev. 58, 267 (1940).

[3] L.D. Landau and E.M. Lifshitz, Fluid Mechanics (Butterworth-Heinemann, Oxford, 1987).

[4] W. Israel and J. M. Stewart, Ann. Phys. 118, 341 (1979).

[5] P. Huovinen and D. Molnar, Phys. Rev. C 79, 014906 (2009).

[6] H. Grad, Comm. Pure Appl. Math. 2, 331 (1949).

[7] G. S. Denicol, T. Koide and D. H. Rischke, Phys. Rev. Lett. 105, 162501 (2010).

[8] A. Jaiswal, Phys. Rev. C 87, 051901 (2013)

[9] J. D. Bjorken, Phys. Rev. D 27, 140 (1983).

[10] A. Muronga, Phys. Rev. C 69, 034903 (2004)

[11] M. Martinez and M. Strickland, Phys. Rev. C 79, 044903
(2009)

[12] A. El, A. Muronga, Z. Xu and C. Greiner, Phys. Rev. C 79, 044914 (2009)

[13] A. Jaiswal, R. S. Bhalerao and S. Pal, Phys. Lett. B 720, 347 (2013)

[14] A. El, Z. Xu and C. Greiner, Phys. Rev. C 81, 041901 (2010).

[15] C. Y. Cha and B. J. McCoy, J. Chem. Phys. 54, 4369 (1971).

[16] S.R. de Groot, W.A. van Leeuwen, and Ch.G. van Weert, Relativistic Kinetic Theory - Principles and Applications (North-Holland, Amsterdam, 1980).

[17] S. Chapman and T. G. Cowling, The Mathematical Theory of Non-Uniform Gases, (Cambridge University Press, Cambridge, 1970), 3rd ed. 
[18] J. L. Anderson and H. R. Witting Physica 74, 466 (1974).

[19] P. Romatschke, Phys. Rev. D 85, 065012 (2012).

[20] G. Baym, Phys. Lett. B 138, 18 (1984).

[21] W. Florkowski, R. Ryblewski and M. Strickland, arXiv:1304.0665 [nucl-th].

[22] A. El, Z. Xu and C. Greiner, Nucl. Phys. A 806, 287 (2008)

[23] Z. Xu and C. Greiner, Phys. Rev. C 71, 064901 (2005);
76, 024911 (2007).

[24] K. Dusling, G. D. Moore and D. Teaney, Phys. Rev. C 81, 034907 (2010)

[25] D. Teaney, Phys. Rev. C 68, 034913 (2003)

[26] R. S. Bhalerao, A. Jaiswal, S. Pal and V. Sreekanth, in preparation. 